\title{
Regularity of Jacobians by subdeterminants
}

\author{
Anna Verde \\ (Communicated by Carlo Sbordone) \\ 2000 Mathematics Subject Classification. Primary 42B25; Secondary \\ 26B10. \\ Keywords and phrases. Regularity of Jacobian, cofactor matrix, Hardy space. \\ Abstract. Some regularity results for the Jacobian determinant $J_{u}$ of a \\ mapping $u \in W^{1, n-1}\left(\Omega, \mathbb{R}^{n}\right)$, having the cofactor matrix of $D u$ in $L^{\frac{n}{n-1}}(\Omega)$, in \\ Hardy-Orlicz spaces are given.
}

\section{Introduction}

Let $\Omega$ be an open subset of $\mathbb{R}^{n}, n \geq 2$. We shall denote by $J_{u}=J(x, u)$ the Jacobian determinant of a mapping $u=\left(u^{1}, \ldots, u^{n}\right): \Omega \rightarrow \mathbb{R}^{n}$ in the Sobolev space $W^{1, n}\left(\Omega, \mathbb{R}^{n}\right)$. The phenomenon of improved integrability for the Jacobian is studied in a series of papers (see [3], [6], [13], [17], [18], [16], etc.).

The first relevant result in the study of the higher integrability property of the Jacobian, given by S. Müller [M1], states that if $u \in W^{1, n}\left(\Omega, \mathbb{R}^{n}\right)$ is an orientation preserving mapping, i.e. $J_{u} \geq 0$, then $J_{u} \log \left(e+J_{u}\right) \in L_{l o c}^{1}(\Omega)$. The result is local.

In [10] the authors investigated what are the minimal assumptions on $u$ under which $J_{u} \log \left(e+J_{u}\right)$ is globally integrable on $\Omega$. 
It is worth pointing out that also in the case when the hypothesis $J_{u} \geq 0$ is removed, the Jacobian determinant enjoys higher regularity. In this context we recall the celebrated result of [5] which states that if $u \in W^{1, n}\left(\mathbb{R}^{n}, \mathbb{R}^{n}\right)$ then $J_{u}$ belongs to the Hardy space $\mathcal{H}^{1}\left(\mathbb{R}^{n}\right)$.

Recently it has been proved that if $u \in W^{1, n-1}\left(\Omega, \mathbb{R}^{n}\right)$ is an orientation preserving mapping and $\left|D^{\#} u\right| \in L^{\frac{n}{n-1}}(\Omega)$, where $D^{\#} u$ denotes the cofactor matrix of $D u$, then $J_{u} \log \left(e+J_{u}\right) \in L_{\text {loc }}^{1}(\Omega)$, see [7], [20]. Moreover in [12] it is shown that under previous assumptions an analogous result of [5] yields, i. e. $J_{u}$ lies in the Hardy space $\mathcal{H}^{1}(\Omega)$ and

$$
\left\|J_{u}\right\|_{\mathcal{H}^{1}(\Omega)} \leq c\left\|D^{\#} u\right\|_{L^{\frac{n}{n-1}}}^{\frac{n}{n-1}} .
$$

It is important to realize that the cofactor matrix $D^{\#} u$ tell us how $n-1$ dimensional objects are deformed under the mapping $f$.

The study of maps in $W^{1, n-1}$ is of great interest in nonlinear elasticity. For example for stored-energy density functions, there exist deformations with finite energy which are not in $W^{1, n}$ (see [2]).

Starting from these results, the aim of this paper is to try to describe some of the properties of the Jacobian determinant in terms of the subdeterminants.

In Section 3, by considering hypotheses on the cofactor matrix $D^{\#} u$, we look for assumptions on $u$ under which $J_{u} \log \left(e+J_{u}\right)$ is globally integrable on $\Omega$ (see Theorem 3.5).

In order to solve this problem if $\Omega$ is a bounded open domain in $\mathbb{R}^{n}$ with strongly Lipschitz boundary $\partial \Omega$, we consider some control on the boundary value of $u$ which gives better integrability of the cofactor matrix $D^{\#} u$.

Next, in the spirit of [16] and [12], we generalize these results to the setting of Orlicz spaces.

The crucial elements in the proofs are the estimate (1.1) and a simple version of Hölder's inequality in Orlicz spaces.

In fact in [10] a div-curl type extension to forms that includes the case $|D u| \in L^{n-1}\left(\Omega, \mathbb{R}^{n \times n}\right),\left|D^{\#} u\right| \in L^{\frac{n-1}{n-2}}(\Omega)$, has been considered. However we point out that here we require less on the integrability of $D^{\#} u$ and in particular we give an estimate on $J_{u}$ only in terms of $D^{\#} u$.

Next in Section 4, we define as a Schwartz distribution the expression $J \log (e+|J|)$, and we give a result concerning a strong convergence of Jacobians locally on $\Omega$ in $L \log L(\Omega)$, the space of all measurable functions $f$ such that

$$
\int_{\Omega}|f| \log (e+|f|) d x<\infty
$$


equipped with the Luxemburg norm that is equivalent to the following nonlinear functional

$$
\int_{\Omega}|f| \log \left(e+\frac{|f|}{\|f\|_{L^{1}(\Omega)}}\right) d x
$$

\section{Preliminaries}

This section is devoted to establishing notation for the technical details associated with the forthcoming calculations. To every pair of ordered $l$ tuples $I=\left(i_{1}, \ldots i_{l}\right)$ and $J=\left(j_{1}, \ldots j_{l}\right)$ with $1 \leq i_{1}<\ldots<i_{l} \leq n$ and $1 \leq j_{1}<\ldots<j_{l} \leq n$ there corresponds an $l \times l$-subdeterminant of the differential matrix denoted by

$$
\frac{\partial u^{I}}{\partial x_{J}}=\frac{\partial\left(u^{i_{1}}, \ldots, u^{i_{l}}\right)}{\partial\left(x_{j_{1}}, \ldots, x_{j_{l}}\right)}
$$

Recall that the volume form $J(x, u) d x$ can be written as the following wedge product

$$
J(x, u) d x=d u^{1} \wedge \ldots \wedge d u^{n}
$$

Let us consider the ordered collection of the partial products of the linear forms $d u^{1}, d u^{2}, \ldots, d u^{n}$

$$
\left\{d u^{i_{1}} \wedge \ldots \wedge d u^{i_{l}}: 1 \leq i_{1}<\ldots<i_{l} \leq n\right\}
$$

Thus, for $l=n$ we obtain the Jacobian $J(x, u)$ and for $l=n-1$ the matrix $D^{\#} u$ of cofactors of $D u$. Let us denote by $\Lambda^{l} \Omega$ the space of $l$-forms on $\Omega$, where $\Omega$ is an open subset of $\mathbb{R}^{n}$. The familiar Cramer's rule for the inverse of a matrix now reads as

$$
D u(x) D^{\#} u(x)=D^{\#} u(x) D u(x)=J(x, u) I
$$

We have Hadamard's inequalities

$$
|J(x, u)| \leq\left|D^{\#} u\right|^{\frac{n}{n-1}} \leq|D u(x)|^{n}
$$

Now we recall some definitions of function spaces. Let us fix a nonnegative radially symmetric function $\phi \in C_{0}^{\infty}\left(\mathbb{R}^{n}\right)$ supported in the unit ball and having integral 1. For each $t>0$, we consider $\phi_{t}(x)=t^{-n} \phi\left(\frac{x}{t}\right)$. Given $h \in L_{l o c}^{1}(\Omega)$, the associated maximal function of $h$ is defined as

$$
\mathcal{M} h(x)=\sup \left\{\left|h * \phi_{t}(x)\right|: 0<t<\operatorname{dist}(x, \partial \Omega)\right\}
$$


The Hardy space $\mathcal{H}^{1}(\Omega)$ consists of distributions $h \in \mathcal{D}^{\prime}(\Omega)$ such that

$$
\|h\|_{\mathcal{H}^{1}(\Omega)}=\|\mathcal{M} h\|_{L^{1}(\Omega)}<+\infty
$$

For $\Omega=\mathbb{R}^{n}$ this definition coincides with the usual one of $\mathcal{H}^{1}\left(\mathbb{R}^{n}\right)$ (see [5]).

Now, recalling that the Hardy-Littlewood maximal operator is defined on $L_{l o c}^{1}(\Omega)$ by the rule

$$
M h(x)=\sup \left\{f_{B(x, t)}|h(y)| d y: 0<t<\operatorname{dist}(x, \partial \Omega)\right\}
$$

we have

$$
\mathcal{M} h(x) \leq c(n) \operatorname{Mh}(x)
$$

Next, let $\mathcal{P}:[0,+\infty) \rightarrow[0,+\infty)$ be a convex function vanishing at 0 . The Orlicz space, denoted by $L^{\mathcal{P}}(\Omega)$, consists of all measurable function $f$ on $\Omega$ such that $\int_{\Omega} \mathcal{P}\left(k^{-1}|f|\right)<\infty$, for some $k=k(f)>0$. We shall make use of the Luxemburg norm

$$
\|f\|_{L^{\mathcal{P}}(\Omega)}=\inf \left\{k>0: \int_{\Omega} \mathcal{P}\left(k^{-1}|f|\right) \leq 1\right\}
$$

so $L^{\mathcal{P}}(\Omega)$ becomes a Banach space.

Definition 2.1. The Hardy-Orlicz space $\mathcal{H}^{\mathcal{P}}(\Omega)$ consists of distributions $h \in \mathcal{D}^{\prime}(\Omega)$ such that

$$
\|h\|_{\mathcal{H}^{\mathcal{P}}(\Omega)}=\|\mathcal{M} h\|_{L^{\mathcal{P}}(\Omega)}<\infty
$$

For more information about Hardy-Orlicz spaces see for instance [12] and [16].

\section{Global higher integrability of Jacobians on bounded domains}

Let us begin this section with the following inequalities

$$
|J(x, u)| \leq c(n) \prod_{j=1}^{n}\left[\left|d u^{1} \wedge \ldots \wedge d u^{j-1} \wedge d u^{j+1} \wedge \ldots \wedge d u^{n}\right|\right]^{\frac{1}{n-1}}
$$

and

$$
\left\|J_{u}\right\|_{\mathcal{H}^{1}(\Omega)} \leq c_{p}(n) \prod_{j=1}^{n}\left\|d u^{1} \wedge \ldots \wedge d u^{j-1} \wedge d u^{j+1} \wedge \ldots \wedge d u^{n}\right\|_{L^{p_{j}}(\Omega)}^{\frac{1}{n-1}}
$$


where $p=\left(p_{1}, \ldots, p_{n}\right)$ is an arbitrary $n$-tuple of exponents $p_{1}, \ldots, p_{n}>1$, such that

$$
\frac{1}{p_{1}}+\ldots+\frac{1}{p_{n}}=n-1
$$

In the sequel, $\Omega$ will denote a bounded open domain in $\mathbb{R}^{n}$ with strongly Lipschitz boundary $\partial \Omega$.

Definition 3.1. Let $\alpha=\left(\alpha_{1}, \ldots, \alpha_{n}\right)$ such that $\alpha_{j} \geq 0$ and $\sum_{j=1}^{n} \alpha_{j}=n$ and let $\phi \in W^{1, n-1}\left(\Omega, \mathbb{R}^{n}\right),\left|D^{\#} \phi\right| \in L^{\frac{n}{n-1}}(\Omega)$. We say that $\phi \in$ $K_{\alpha}\left(\partial \Omega, \mathbb{R}^{n}\right)$ if there exists $v \in W^{1, n-1}\left(\Omega, \mathbb{R}^{n}\right)$ so that $d v^{1} \wedge \ldots \wedge d v^{j-1} \wedge$ $d v^{j+1} \wedge \ldots \wedge d v^{n} \in L^{\frac{n}{n-1}} \log ^{\alpha_{j}} L(\Omega)$, for $j=1,2, \ldots n$ and $v_{/ \partial \Omega}=\phi$

We define $\forall j=1, \ldots, n$ the following functional on $K_{\alpha}$

$$
\begin{gathered}
{\left[d \phi^{1} \wedge \ldots \wedge d \phi^{j-1} \wedge d \phi^{j+1} \wedge \ldots \wedge d \phi^{n}\right]_{\alpha_{j}}} \\
(3.3)=\inf \left\{\left\|d v^{1} \wedge \ldots \wedge d v^{j-1} \wedge d v^{j+1} \wedge \ldots \wedge d v^{n}\right\|_{L^{\frac{n}{n-1}} \log ^{\alpha_{j}} L(\Omega)}: v_{/ \partial \Omega}=u\right\}
\end{gathered}
$$

Before coming to the main theorem of this section we introduce some more function spaces.

One space is

$$
\mathcal{H}_{z}^{1}(\Omega):=\left\{f \in L^{1}(\Omega) ; f_{z} \in \mathcal{H}^{1}\left(\mathbb{R}^{n}\right)\right\}
$$

where $f_{z}$ is the zero extension of $f$ to $\mathbb{R}^{n}$.

Every function $f \in \mathcal{H}_{z}^{1}(\Omega)$ satisfies $\int_{\Omega} f(x) d x=0$. The space obtained by removing this cancellation condition is

$$
h_{z}^{1}(\Omega)=\left\{f \in L^{1}(\Omega) ; f-f_{\Omega} f(x) d x \in \mathcal{H}_{z}^{1}(\Omega)\right\}
$$

Norms on these spaces are defined in the obvious way:

$$
\begin{gathered}
\|f\|_{\mathcal{H}_{z}^{1}(\Omega)}=\left\|f_{z}\right\|_{\mathcal{H}^{1}\left(\mathbb{R}^{n}\right)} \\
\|f\|_{h_{z}^{1}(\Omega)}=\left\|f-\frac{1}{\Omega} \int_{\Omega} f(x)\right\|_{\mathcal{H}_{z}^{1}(\Omega)}+\left|f_{\Omega} f(x)\right|
\end{gathered}
$$

In the sequel we will need the following results.

Lemma 3.2. [10]

i) Suppose $f \in L \log L(\Omega)$ and $\int_{\Omega} f(x) d x=0$. Then $f \in \mathcal{H}_{z}^{1}(\Omega)$ and $\|f\|_{\mathcal{H}_{z}^{1}(\Omega)} \leq c\|f\|_{L \log L(\Omega)}$. 
ii) $L \log L(\Omega) \subset h_{z}^{1}(\Omega)$ with $\|f\|_{h_{z}^{1}(\Omega)} \leq c\|f\|_{L \log L(\Omega)}$.

Proposition 3.3. [10] Suppose $f \in h_{z}^{1}(\Omega)$ and $f \geq 0$ on $\Omega$. Then $f \in L \log L(\Omega)$ and

$$
\|f\|_{L \log L(\Omega)} \leq c\|f\|_{h_{z}^{1}(\Omega)}
$$

The main result of this Section is

Theorem 3.4. Suppose $u \in W^{1, n-1}\left(\Omega, \mathbb{R}^{n}\right), D^{\#} u \in L^{\frac{n}{n-1}}(\Omega), u \in$ $K_{\alpha}\left(\partial \Omega, \mathbb{R}^{n}\right)$. Then $J_{u} \in h_{z}^{1}(\Omega)$ and

$$
\begin{aligned}
\left\|J_{u}\right\|_{h_{z}^{1}(\Omega)} \leq & c \prod_{j=1}^{n}\left\{\left\|d u^{1} \wedge \ldots \wedge d u^{j-1} \wedge d u^{j+1} \wedge \ldots \wedge d u^{n}\right\|_{L^{\frac{n}{n-1}}(\Omega)}^{\frac{1}{n-1}}\right. \\
& \left.+\left[d u^{1} \wedge \ldots \wedge d u^{j-1} \wedge d u^{j+1} \wedge \ldots \wedge d u^{n}\right]_{\alpha_{j}}^{\frac{1}{n-1}}\right\}
\end{aligned}
$$

Proof. Since $\Omega$ has a strongly Lipschitz boundary, we can extend $u$ to $\mathbb{R}^{n}$ so that

$$
\begin{aligned}
\| d u^{1} & \wedge \ldots \wedge d u^{j-1} \wedge d u^{j+1} \wedge \ldots \wedge d u^{n} \|_{L^{\frac{n}{n-1}}\left(\mathbb{R}^{n}\right)} \\
& \leq c\left\|d u^{1} \wedge \ldots \wedge d u^{j-1} \wedge d u^{j+1} \wedge \ldots \wedge d u^{n}\right\|_{L^{\frac{n}{n-1}}(\Omega)}
\end{aligned}
$$

for each $j$ (see [14] for more details).

By our assumptions, we can choose $v \in W^{1, n-1}\left(\mathbb{R}^{n}, \mathbb{R}^{n}\right)$ such that

$$
\begin{aligned}
\| d v^{1} & \wedge \ldots \wedge d v^{j-1} \wedge d v^{j+1} \wedge \ldots \wedge d v^{n} \|_{L^{\frac{n}{n-1}} \log ^{\alpha_{j}} L(\Omega)} \\
& \leq c\left[d u^{1} \wedge \ldots \wedge d u^{j-1} \wedge d u^{j+1} \wedge \ldots \wedge d u^{n}\right]_{\alpha_{j}}
\end{aligned}
$$

and $v_{j}=u_{j}$ on $\mathbb{R}^{n} \backslash \Omega, \forall j=1, \ldots, n$. Note that

$$
\begin{aligned}
\| d v^{1} \wedge & \ldots \wedge d v^{j-1} \wedge d v^{j+1} \wedge \ldots \wedge d v^{n} \|_{L^{\frac{n}{n-1}}\left(\mathbb{R}^{n}\right)} \\
\leq & \left\|d u^{1} \wedge \ldots \wedge d u^{j-1} \wedge d u^{j+1} \wedge \ldots \wedge d u^{n}\right\|_{L^{\frac{n}{n-1}}\left(\mathbb{R}^{n}\right)} \\
& +\left\|d v^{1} \wedge \ldots \wedge d v^{j-1} \wedge d v^{j+1} \wedge \ldots \wedge d v^{n}\right\|_{L^{\frac{n}{n-1}}(\Omega)} \\
\leq & c\left(\left\|d u^{1} \wedge \ldots \wedge d u^{j-1} \wedge d u^{j+1} \wedge \ldots \wedge d u^{n}\right\|_{L^{\frac{n}{n-1}}(\Omega)}\right. \\
& \left.+\left[d u^{1} \wedge \ldots \wedge d u^{j-1} \wedge d u^{j+1} \wedge \ldots \wedge d u^{n}\right]_{\alpha_{j}}\right)
\end{aligned}
$$


Next using (3.2) with $p_{1}=p_{2}=\ldots=p_{n}=\frac{n}{n-1},(3.10)$ and (3.11) we obtain

$$
\begin{aligned}
& \left\|J_{u}-J_{v}\right\|_{\mathcal{H}^{1}\left(\mathbb{R}^{n}\right)} \\
& \leq c \prod_{j=1}^{n}\left\|d u^{1} \wedge \ldots \wedge d u^{j-1} \wedge d u^{j+1} \wedge \ldots \wedge d u^{n}\right\|_{L^{\frac{n}{n-1}}\left(\mathbb{R}^{n}\right)}^{\frac{1}{n-1}} \\
& \quad+c \prod_{j=1}^{n}\left\|d v^{1} \wedge \ldots \wedge d v^{j-1} \wedge d v^{j+1} \wedge \ldots \wedge d v^{n}\right\|_{L^{\frac{n}{n-1}}}^{\frac{1}{n-1}}\left(\mathbb{R}^{n}\right) \\
& \leq c \prod_{j=1}^{n}\left\|d u^{1} \wedge \ldots \wedge d u^{j-1} \wedge d u^{j+1} \wedge \ldots \wedge d u^{n}\right\|_{L^{\frac{n}{n-1}}(\Omega)}^{\frac{1}{n-1}} \\
& \left.\quad+c \prod_{j=1}^{n} d u^{1} \wedge \ldots \wedge d u^{j-1} \wedge d u^{j+1} \wedge \ldots \wedge d u^{n}\right]_{\alpha_{j}}^{\frac{1}{n-1}}
\end{aligned}
$$

Let us observe that

$$
J_{u}-J_{v}=\sum_{k=1}^{n} d u^{1} \wedge \ldots \wedge\left(d u^{k}-d v^{k}\right) \wedge \ldots \wedge d v^{n}
$$

Since $u_{k}-v_{k}=0$ outside $\Omega$, the support of $d u^{1} \wedge \ldots \wedge\left(d u^{k}-d v^{k}\right) \wedge \ldots \wedge d v^{n}$ is contained in $\bar{\Omega}$. Thus $J_{u}-J_{v} \in \mathcal{H}_{z}^{1}(\Omega)$ and

$$
\begin{gathered}
\left\|J_{u}-J_{v}\right\|_{h_{z}^{1}(\Omega)}=\left\|J_{u}-J_{v}\right\|_{\mathcal{H}_{z}^{1}(\Omega)}=\left\|J_{u}-J_{v}\right\|_{\mathcal{H}^{1}\left(\mathbb{R}^{n}\right)} \\
\leq c \prod_{j=1}^{n}\left\|d u^{1} \wedge \ldots \wedge d u^{j-1} \wedge d u^{j+1} \wedge \ldots \wedge d u^{n}\right\|_{L^{\frac{n}{n-1}}(\Omega)}^{\frac{1}{n-1}} \\
\quad+c\left[d u^{1} \wedge \ldots \wedge d u^{j-1} \wedge d u^{j+1} \wedge \ldots \wedge d u^{n}\right]_{\alpha_{j}}^{\frac{1}{n-1}}
\end{gathered}
$$

Now we consider $J_{v}$. By (3.1) and Hölder's inequality we have

$$
\begin{aligned}
& \left\|J_{v}\right\|_{h_{z}^{1}(\Omega)} \leq c\left\|J_{v}\right\|_{L \log L(\Omega)} \\
& \quad \leq c \prod_{j=1}^{n}\left\|d v^{1} \wedge \ldots \wedge d v^{j-1} \wedge d v^{j+1} \wedge \ldots \wedge d v^{n}\right\|_{L^{\frac{n}{n-1}} \log ^{\alpha_{j}} L(\Omega)}^{\frac{1}{n-1}} \\
& \quad \leq c \prod_{j=1}^{n}\left[d u^{1} \wedge \ldots \wedge d u^{j-1} \wedge d u^{j+1} \wedge \ldots \wedge d u^{n}\right]_{\alpha_{j}}^{\frac{1}{n-1}}
\end{aligned}
$$

Combining estimates (3.15) and (3.16) we complete the proof.

The next result is the global higher integrability of the Jacobian on $\Omega$. 
Theorem 3.5. Under the same hypotheses of Theorem 3.4, if moreover $J_{u} \geq 0$ then $J_{u} \in L \log L(\Omega)$ and

$$
\begin{aligned}
\left\|J_{u}\right\|_{L \log L(\Omega)} \leq & c \prod_{j=1}^{n}\left\{\left\|d u^{1} \wedge \ldots \wedge d u^{j-1} \wedge d u^{j+1} \wedge \ldots \wedge d u^{n}\right\|_{L^{\frac{n}{n-1}}(\Omega)}^{\frac{1}{n-1}}\right. \\
& \left.+\left[d u^{1} \wedge \ldots \wedge d u^{j-1} \wedge d u^{j+1} \wedge \ldots \wedge d u^{n}\right]_{\alpha_{j}}^{\frac{1}{n-1}}\right\} .
\end{aligned}
$$

This is an immediate consequence of Theorem 3.4 and the Proposition $3.3[10]$.

\section{Nonlinear expressions of Jacobians as Schwartz distributions}

Given $u=\left(u^{1}, \ldots, u^{n}\right): \Omega \rightarrow \mathbb{R}^{n}$ a mapping of Sobolev class, in this section we interpret as Schwartz distributions the two non linear expressions

$$
\begin{gathered}
J_{u} \log \left(e+\left|D^{\#} u\right|\right) \\
J_{u} \log \left(e+\left|J_{u}\right|\right)
\end{gathered}
$$

We start with (4.1).

Let us consider the Zygmund space $L \log L$, of all measurable functions $f$ such that

$$
[f]_{\log }=\int_{\Omega}|f| \log (e+|f|) d x<\infty
$$

The nonlinear functional

$$
\|f\|_{\log }=\inf \left\{k>0:\left[\frac{f}{k}\right]_{\log } \leq 1\right\}
$$

defines a metric on this space.

If $u$ is a mapping in $W^{1, n-1}\left(\Omega, \mathbb{R}^{n}\right)$ such that $\left|D^{\#} u\right| \in L_{l o c}^{\frac{n}{n-1}}$, the two expressions in (4.1), and (4.2) need not be locally integrable. However, we can interpret them as Schwartz distributions on $\Omega$.

Theorem 4.1. it For any sequence $\left(u_{j}\right)$ in $C_{0}^{\infty}\left(\Omega, \mathbb{R}^{n}\right)$ converging to $u$ in $W^{1, n-1}\left(\Omega, \mathbb{R}^{n}\right)$ such that $D^{\#} u_{j}$ converges to $D^{\#} u$ in $L_{l o c}^{\frac{n}{n-1}}$, we have

$$
\lim _{j} \int_{\Omega} \eta J_{u_{j}} \log \left(e+\frac{\left|J_{u_{j}}\right|}{\left\|J_{u_{j}}\right\|}\right)=\left\langle\eta, J_{u} \log \left(e+\frac{\left|J_{u}\right|}{\left\|J_{u}\right\|_{1}}\right)\right\rangle
$$

where the angular brackets designate the value of the distribution at the test function $\eta \in C_{0}^{\infty}(\Omega)$ 
This result can be proved in much the same way as in [15]. In fact from Theorem 4 in [15] the only point remaining concerns the $\mathcal{H}^{1}$ convergence of the Jacobian. To this aim we can prove the following estimate.

Proposition 4.2. For any $u, v \in W^{1, n-1}\left(\Omega, \mathbb{R}^{n}\right)$, with $D^{\#} u, D^{\#} v \in$ $L_{\text {loc }}^{\frac{n}{n-1}}$, we have

$\left\|J_{u}-J_{v}\right\|_{\mathcal{H}^{1}} \leq C(n)\left\{\|D u\|_{n-1}\left\|D^{\#} u-D^{\#} v\right\|_{\frac{n}{n-1}}+\|D u-D v\|_{n-1}\left\|D^{\#} v\right\|_{\frac{n}{n-1}}\right\}$.

Proof. First of all let us observe that we can write

$J_{u}-J_{v}=d u^{1} \wedge\left(d u^{2} \wedge \cdots \wedge d u^{n}-d v^{2} \wedge \cdots \wedge d v^{n}\right)+\left(d u^{1}-d v^{1}\right) \wedge d v^{2} \wedge \cdots \wedge d v^{n}$

Let us consider $\phi_{t}(x)$ like in $(2.2)$, where $x \in \Omega$ and $0<t<\operatorname{dist}(x, \partial \Omega)$. We have

$$
\begin{array}{rl}
\phi_{t} & *\left(J_{u}-J_{v}\right)(x) \\
= & \int_{B(x, t)} \frac{1}{t^{n+1}} u^{1}(y) d \phi\left(\frac{x-y}{t}\right) \wedge\left(d u^{2} \wedge \cdots \wedge d u^{n}-d v^{2} \wedge \cdots \wedge d v^{n}\right) d y \\
& \quad+\int_{B(x, t)} \frac{1}{t^{n+1}}\left(u^{1}-u^{1}\right)(y) d \phi\left(\frac{x-y}{t}\right) \wedge\left(d v^{2} \wedge \cdots \wedge d v^{n}\right)(y) d y
\end{array}
$$

where $B(x, t)$ is the ball centered at $x$ of radius $t$. Until further notice we assume for semplicity that $\Omega$ is a cube. Thus, in particular $B(x, t) \subset$ $Q(x, t) \subset \Omega$. Next we use Hölder's inequality with $s$ and $s_{1}=\frac{s}{s-1}$, where $n<s<n^{2}-n$, to deduce

$$
\begin{aligned}
& \left|\phi_{t} *\left(J_{u}-J_{v}\right)(x)\right| \\
& \leq C\left(f_{Q(x, t)}\left|u^{1}\right|^{s}\right)^{\frac{1}{s}}\left(f_{Q(x, t)}\left|d u^{2} \wedge \cdots \wedge d u^{n}-d v^{2} \wedge \cdots \wedge d v^{n}\right|^{s_{1}}\right)^{\frac{1}{s_{1}}} \\
& \quad+C\left(f_{Q(x, t)}\left|u^{1}-v^{1}\right|^{s}\right)^{\frac{1}{s}}\left(f_{Q(x, t)}\left|d v^{2} \wedge \cdots \wedge d v^{n}\right|^{s_{1}}\right)^{\frac{1}{s_{1}}} .
\end{aligned}
$$

Hence we have the following estimate

$$
\begin{aligned}
\left\|J_{u}-J_{v}\right\|_{\mathcal{H}^{1}} \leq & C(n) \int_{\Omega}\left[M\left(u^{1}\right)^{s}\right]^{\frac{1}{s}}\left[M\left(d u^{2} \wedge \cdots \wedge d u^{n}-d v^{2} \wedge \cdots \wedge d v^{n}\right)^{s_{1}}\right]^{\frac{1}{s_{1}}} \\
& +C(n) \int_{\Omega}\left[M\left(u^{1}-v^{1}\right)^{s}\right]^{\frac{1}{s}}\left[M\left(d v^{2} \wedge \cdots \wedge d v^{n}\right)^{s_{1}}\right]^{\frac{1}{s_{1}}}
\end{aligned}
$$


By Holder's inequality we have

$$
\begin{aligned}
\left\|J_{u}-J_{v}\right\|_{\mathcal{H}^{1}} \leq & C(n)\left(\int_{\Omega}\left[M\left(u^{1}\right)^{s}\right]\right)^{\frac{1}{s}}\left(\int_{\Omega}\left[M\left(d u^{2} \wedge \cdots \wedge d u^{n}-d v^{2} \wedge \cdots \wedge d v^{n}\right)^{s_{1}}\right]\right)^{\frac{1}{s_{1}}} \\
& +C(n)\left(\int_{\Omega}\left[M\left(u^{1}-v^{1}\right)^{s}\right]\right)^{\frac{1}{s}}\left(\int_{\Omega}\left[M\left(d v^{2} \wedge \cdots \wedge d v^{n}\right)^{s_{1}}\right]\right)^{\frac{1}{s_{1}}}
\end{aligned}
$$

Finally we can use the boundedness of the maximal function in the spaces $L^{\frac{n^{2}-n}{s}}$ and $L^{\frac{n}{s_{1}(n-1)}}$ and then the Sobolev's imbedding Theorem to complete the proof.

We conclude this section with a result concerning the convergence of Jacobians. To this aim the following Lemma will be useful.

Lemma 4.3. For each $f \in L \log L(\Omega)$ with $f \geq 0$, there exists a constant $c>1$ such that

$$
\|f\|_{L \log L(\Omega)} \leq \int_{\Omega} f \log \left(e+\frac{f}{\|f\|_{L^{1}(\Omega)}}\right) \leq c\|f\|_{L \log L(\Omega)} .
$$

Theorem 4.4. Given $\left\{u_{k}\right\}$ a sequence of orientation preserving mappings $u_{k}: \Omega \rightarrow \mathbb{R}^{n}$ converging to $u$ in $W^{1, n-1}\left(\Omega, \mathbb{R}^{n}\right)$, such that $D^{\#} u_{k} \rightarrow D^{\#} u$ in $L^{\frac{n}{n-1}}$. Then

$$
\lim _{k} \int_{E}\left|J_{u_{k}}-J_{u}\right| \log \left(e+\frac{\left|J_{u_{k}}-J_{u}\right|}{\left\|J_{u_{k}}-J_{u}\right\|_{L^{1}(E)}}\right) d x=0
$$

for each compact $E \subset \Omega$.

Proof. The proof is similar in the spirit to the proof of Theorem 6 in [15]. For the sake of clarity let us give the main ideas of the proof. Fix $E \subset \Omega$ compact subset, by Proposition ?? and Theorem 1 in [15] we have

$$
\lim _{k} \int_{\Omega} \eta J_{u_{k}} \log \left(e+J_{u_{k}}\right) d x=\int_{\Omega} \eta J_{u} \log \left(e+J_{u}\right) d x
$$

where $\eta \in C_{0}^{\infty}$ is an arbitrary nonnegative function equal to 1 on $E \subset \Omega$. Taking infimum with respect to all such functions $\eta$ yields

$$
\lim _{k} \int_{E} J_{u_{k}} \log \left(e+J_{u_{k}}\right) d x=\int_{E} J_{u} \log \left(e+J_{u}\right) d x
$$

Let us denote

$$
\bar{J}_{k}=\max \left\{J_{u}, J_{u_{k}}\right\}, \quad \underline{J}_{k}=\min \left\{J_{u}, J_{u_{k}}\right\} .
$$


Then

$$
J_{u_{k}} \log \left(e+J_{u_{k}}\right)-J_{u} \log \left(e+J_{u}\right)=
$$

$$
\bar{J}_{k} \log \left(e+\bar{J}_{k}\right)-J_{u} \log \left(e+J_{u}\right)+\underline{J}_{k} \log \left(e+\underline{J}_{k}\right)-J_{u} \log \left(e+J_{u}\right) .
$$

By Dominated Convergence theorem

$$
\lim _{k \rightarrow \infty} \int_{E} \underline{J}_{k} \log \left(e+\underline{J}_{k}\right)=\int_{E} J_{u} \log \left(e+J_{u}\right) .
$$

From (4.8), (4.9) and (4.10) we deduce

$$
\lim _{k \rightarrow \infty} \int_{E} \bar{J}_{k} \log \left(e+\bar{J}_{k}\right)=\int_{E} J_{u} \log \left(e+J_{u}\right)
$$

For $0<y \leq x$ by the elementary inequality

$$
(x-y) \log (e+x-y) \leq x \log (e+x)-y \log (e+y),
$$

using (4.11) it follows that

$\lim _{k \rightarrow \infty} \int_{E}\left(\bar{J}_{k}-J_{u}\right) \log \left(e+\bar{J}_{k}-J_{u}\right) \leq \lim _{k \rightarrow \infty}\left(\int_{E} \bar{J}_{k} \log \left(e+\bar{J}_{k}\right)-\int_{E} J_{u} \log \left(e+J_{u}\right)\right)=0$

and

$\lim _{k \rightarrow \infty} \int_{E}\left(\underline{J}_{k}-J_{u}\right) \log \left(e+\underline{J}_{k}-J_{u}\right) \leq \lim _{k \rightarrow \infty}\left(\int_{E} J_{u} \log \left(e+J_{u}\right)-\int_{E} \bar{J}_{k} \log \left(e+\bar{J}_{k}\right)\right)=0$

Therefore we obtain

$$
\lim _{k \rightarrow \infty} \int_{E}\left|J_{u_{k}}-J_{u}\right| \log \left(e+\left|J_{u_{k}}-J_{u}\right|\right)=0
$$

On the other hand, by the hypotheses, applying Theorem (2.1) in [7], we find a constant $C$ such that

$$
\left\|J_{u_{k}}\right\|_{L \log L(E)} \leq C
$$

and, moreover, there exists a subsequence $J_{u_{k_{l}}}$ converging to $J_{u}$ almost everywhere. Then we can prove

$$
\lim _{k}\left\|J_{u_{k}}-J_{u}\right\|_{L^{1}(E)}=0
$$


Now let us observe that from (4.6)

$$
\begin{aligned}
\left\|J_{u_{k}}-J_{u}\right\|_{L \log L(E)} \leq & \int_{E}\left|J_{u_{k}}-J_{u}\right| \log \left(e+\frac{\left|J_{u_{k}}-J_{u}\right|}{\left\|J_{u_{k}}-J_{u}\right\|_{L^{1}(E)}}\right) \\
\leq & \int_{E}\left|J_{u_{k}}-J_{u}\right| \log \left(e+\left|J_{u_{k}}-J_{u}\right|\right) \\
& \quad+\left\|J_{u_{k}}-J_{u}\right\|_{L^{1}(E)} \log \left(1+\frac{1}{\left\|J_{u_{k}}-J_{u}\right\|_{L^{1}(E)}}\right)
\end{aligned}
$$

Combining (4.12), (4.13), (4.14) we complete the proof.

\section{Appendix}

This section is devoted to extend the results of Section 3 to Hardy-Orlicz spaces. For the convenience of the reader we repeat the relevant material from Section 3 without proofs, thus making our exposition self-contained.

We begin with a brief review of maximal inequalities in Orlicz spaces.

Lemma 5.1. [6] Given an Orlicz function $\mathcal{P}:[0, \infty) \rightarrow[0, \infty)$ such that $\int_{0}^{1} s^{-2} \mathcal{P}(s) d s<\infty$ we define

$$
\mathcal{R}(t)=\mathcal{P}(t)+t \int_{0}^{t} s^{-2} \mathcal{P}(s) d s
$$

Then every locally integrable function $h$ on a cube $\Omega \subset \mathbb{R}^{n}$ satisfies

$$
\|h\|_{L^{\mathcal{R}}(\Omega)} \leq\left(1+2^{n}\right)\|M(h)\|_{L^{\mathcal{P}}(\Omega)}
$$

and

$$
\int_{\Omega} \mathcal{P}(M(h)) \leq 2 \cdot 3^{n} \int_{\Omega} \mathcal{R}(2 h)
$$

whenever the right hand side is finite. If the function $t \rightarrow t^{-p} \mathcal{P}(t)$ is increasing for some $p>1$, then $\mathcal{P}(t) \leq \mathcal{R}(t) \leq \frac{p}{p-1} \mathcal{P}(t)$ and we have

$$
\|M(h)\|_{L^{\mathcal{P}}(\Omega)} \leq \frac{4 \cdot 3^{n} p}{p-1}\|h\|_{L^{\mathcal{P}}(\Omega)}
$$

Next result concerns the integrability of Jacobians in Hardy-Orlicz spaces.

Theorem 5.2. Let $\mathcal{P}:[0, \infty) \rightarrow[0, \infty)$ be a Young function such that $t^{-N} \mathcal{P}(t)$ decreases to zero for some $N>1$. Consider a mapping $u: \Omega \rightarrow \mathbb{R}^{n}$ of the Sobolev class $W^{1, n-1}\left(\Omega, \mathbb{R}^{n}\right)$, with $\left|D^{\#} u\right| \in L^{\mathcal{P}}(\Omega)$. Then its Jacobian 
belongs to the Hardy-Orlicz space $\mathcal{H}^{\mathcal{P}}(\Omega)$, and we have

$$
\left\|J_{u}\right\|_{\mathcal{H}^{\mathcal{P}}(\Omega)} \leq c_{\mathcal{P}}(n)\left\|\left|D^{\#} u\right|^{\frac{n}{n-1}}\right\|_{L^{\mathcal{P}}(\Omega)} .
$$

In analogy with the definitions given in Section 3 we define

$$
h_{z}^{\mathcal{P}}(\Omega)=\left\{f \in L^{1}(\Omega) ; f-f_{\Omega} f(x) d x \in \mathcal{H}_{z}^{\mathcal{P}}(\Omega)\right\}
$$

where

with

$$
\mathcal{H}_{z}^{\mathcal{P}}(\Omega):=\left\{f \in L^{1}(\Omega) ; f_{z} \in \mathcal{H}^{\mathcal{P}}\left(\mathbb{R}^{n}\right)\right\}
$$

$$
\|f\|_{h_{z}^{\mathcal{P}}(\Omega)}=\left\|f-\frac{1}{\Omega} \int_{\Omega} f(x)\right\|_{\mathcal{H}_{z}^{\mathcal{P}}(\Omega)}+\left|f_{\Omega} f(x)\right|
$$

From Lemma 5.1 it is easy to prove the following results

Proposition 5.3. Suppose $f \in h_{z}^{\mathcal{P}}(\Omega)$ and $f \geq 0$ on $\Omega$. Then $f \in L^{\mathcal{R}}(\Omega)$ and

$$
\|f\|_{L^{\mathcal{R}}(\Omega)} \leq c\|f\|_{h_{z}^{\mathcal{P}}(\Omega)}
$$

where $\mathcal{P}$ is like in Lemma 5.1 and $\mathcal{R}$ is defined in (5.1).

Lemma 5.4. Suppose $f \in L^{\mathcal{R}}(\Omega)$ and $\int_{\Omega} f(x) d x=0$. Then $f \in h_{z}^{\mathcal{P}}(\Omega)$ and $\|f\|_{\mathcal{H}_{z}^{\mathcal{P}}(\Omega)} \leq c\|f\|_{L^{\mathcal{R}}(\Omega)}$. However, $L^{\mathcal{R}}(\Omega) \subset h_{z}^{\mathcal{P}}(\Omega)$ with $\|f\|_{h_{z}^{\mathcal{P}}(\Omega)} \leq$ $c\|f\|_{L^{\mathcal{R}}(\Omega)}$, where $\mathcal{P}$ is like in Lemma 5.1 and $\mathcal{R}$ is defined in (5.1).

Lemma 5.5. Suppose $\mathcal{R}, \mathcal{R}_{i}:[0, \infty) \rightarrow[0, \infty) i=1, \ldots n$ are continuous, monotone increasing functions for which there exist positive constants $c$ and $d$ such that

i) $\mathcal{R}_{1}^{-1}(t) \ldots \mathcal{R}_{n}^{-1}(t) \leq c \mathcal{R}^{-1}(t)$ for all $t>0$, and

ii) $\mathcal{R}\left(\frac{t}{d}\right) \leq \frac{1}{2} \mathcal{R}(t)$ for all $t>0$.

Suppose also that $\Omega$ is an open subset of $\mathbb{R}^{n}, f_{i} \in L^{\mathcal{R}_{i}}(\Omega), \forall i=1, \ldots n$. Then $f=f_{1} \ldots f_{n} \in L^{\mathcal{R}}(\Omega)$ and

$$
\|f\|_{L^{\mathcal{R}}} \leq C \prod_{i=1}^{n}\|f\|_{L^{\mathcal{R}_{i}}}
$$

By induction applied to Theorem A.1 [10] the proof comes soon.

Definition 5.6. Let $\mathcal{P}:[0, \infty) \rightarrow[0, \infty)$ be a Young function such that $t^{-N} \mathcal{P}(t)$ decreases to zero for some $N>1$. Define $\mathcal{R}:[0, \infty) \rightarrow[0, \infty)$ like in Lemma 5.1. We say that $\phi \in W^{1, n-1}\left(\Omega, \mathbb{R}^{n}\right)$ with $\left|D^{\#} \phi\right| \in$ $L^{\mathcal{P}}(\Omega)$ belongs to $K_{\mathcal{R}}\left(\partial \Omega, \mathbb{R}^{n}\right)$ if there exists $v \in W^{1, n-1}\left(\Omega, \mathbb{R}^{n}\right)$ so that $d v^{1} \wedge \ldots \wedge d v^{j-1} \wedge d v^{j+1} \wedge \ldots \wedge d v^{n} \in L^{\mathcal{R}_{j}}(\Omega), j=1,2, \ldots n$ and $v_{/ \partial \Omega}=\phi$ (where $\mathcal{R}_{j} j=1,2, \ldots n$ are related with the function $\mathcal{R}$ like in Lemma 5.5. 
We define the following functional on $K_{\mathcal{R}}$

$$
\begin{aligned}
& {\left[d \phi^{1} \wedge \ldots \wedge d \phi^{j-1} \wedge d \phi^{j+1} \wedge \ldots \wedge d \phi^{n}\right]_{\mathcal{R}_{j}}} \\
& \quad=\inf \left\{\left\|d v^{1} \wedge \ldots \wedge d v^{j-1} \wedge d v^{j+1} \wedge \ldots \wedge d v^{n}\right\|_{L^{\mathcal{R}_{j}(\Omega)}}: v_{/ \partial \Omega}=u\right\}
\end{aligned}
$$

From the results above, the proofs of the next two theorems follow the same arguments of Theorems 3.4, 3.5.

Theorem 5.7. Suppose $u \in W^{1, n-1}\left(\Omega, \mathbb{R}^{n}\right),\left|D^{\#} u\right| \in L^{\mathcal{P}}(\Omega), u_{/ \partial \Omega} \in$ $K_{\mathcal{R}}\left(\partial \Omega, \mathbb{R}^{n}\right)$, where the functions $\mathcal{P}$ and $\mathcal{R}$ are like in the Definition 5.6. Then $J_{u} \in h_{z}^{\mathcal{P}}(\Omega)$ and

$$
\begin{aligned}
\left\|J_{u}\right\|_{h_{z}^{\mathcal{P}}(\Omega)} \leq & c \prod_{j=1}^{n}\left\{\left\|d u^{1} \wedge \ldots \wedge d u^{j-1} \wedge d u^{j+1} \wedge \ldots \wedge d u^{n}\right\|_{L^{\mathcal{P}}(\Omega)}^{\frac{1}{n-1}}\right. \\
& \left.+\left[d u^{1} \wedge \ldots \wedge d u^{j-1} \wedge d u^{j+1} \wedge \ldots \wedge d u^{n}\right]_{\mathcal{R}_{j}}^{\frac{1}{n-1}}\right\} .
\end{aligned}
$$

Theorem 5.8. In the same hypotheses of Theorem 5.7, if moreover $J_{u} \geq 0$ then $J_{u} \in L^{\mathcal{R}}(\Omega)$ and

$$
\begin{aligned}
\left\|J_{u}\right\|_{L^{\mathcal{R}}(\Omega)} \leq & c \prod_{j=1}^{n}\left\{\left\|d u^{1} \wedge \ldots \wedge d u^{j-1} \wedge d u^{j+1} \wedge \ldots \wedge d u^{n}\right\|_{L^{\mathcal{P}}(\Omega)}^{\frac{1}{n-1}}\right. \\
& \left.+\left[d u^{1} \wedge \ldots \wedge d u^{j-1} \wedge d u^{j+1} \wedge \ldots \wedge d u^{n}\right]_{\mathcal{R}_{j}}^{\frac{1}{n-1}}\right\} .
\end{aligned}
$$

\section{References}

[1] R. A. Adams, Sobolev spaces, Academic Press, New York, 1975.

[2] J. M. Ball, Convexity Conditions and Existence Theorems in Nonlinear Elasticity, Arch. Rat. Mech. Anal., 63 (1977), 337-403.

[3] H. Brézis, N. Fusco and C. Sbordone, Integrability for the Jacobian of orientation preserving mappings, J. Funct. Anal., 115 (2) (1993) $425-431$.

[4] D.-C. Chang, S. G. Krantz and E. M. Stein, $\mathcal{H}^{p}$ Theory on a smooth domain in $\mathbb{R}^{n}$ and elliptic boundary problems, J. Funct. Anal., 114 (1993) 286-347.

[5] R. Coifman, P. L. Lions, Y. Meyer and S. Semmes, Compensated compactness and Hardy spaces, J. Math. Pures Appl., (1993), 247286. 
[6] L. Greco, T. Iwaniec and G. Moscariello, Limits on the improved integrability of the volume forms, Indiana Univ. Math. J., 44 (1995) 305-339.

[7] F. Giannetti, T. Iwaniec, J. Onninen and A. Verde, Estimates of Jacobians by Subdeterminants, J. Geom. Anal., 12(2002) (2), 223-254.

[8] L. Greco, T. Iwaniec, C. Sbordone and B. Stroffolini, Degree formulas for maps with nonintegrable Jacobian, Topol. Methods Nonlinear Anal., 6(1995) (1), 81-95.

[9] L. Greco and A. Verde, On some nonlinear expressions of Jacobians, Differential and Integral Equations, 13(10-12) (Oct-Dec 2000), 15691582 .

[10] J. Hogan, C. Li, A. McIntosh and K. Zhang, Global higher integrability of Jacobians on bounded domains, Ann. Inst. H. Poincaré, Analyse non linéaire, 17(2) (2000), 193-217.

[11] T. Iwaniec, Integrability theory of the Jacobians, Vorlesungsreihe Rheinische Friedrich-Wilhelms-Universität Bonn, 36(1995).

[12] T. Iwaniec and J. Onninen, $\mathcal{H}^{1}$-Estimates of Jacobians by Subdeterminants, Math. Ann., 324(2002) (2), 341-358.

[13] T. Iwaniec and C. Sbordone, On the integrability of the Jacobian under minimal hypotheses, Arch. Rational Mech. Anal., 119 (1992), 129-143.

[14] T. Iwaniec, C. Scott and B. Stroffolini, Nonlinear Hodge theory on manifolds with Boundary, Annali di Matematica Pura ed Applicata (IV), CL XXV(1999), 177(1999), 37-115.

[15] T. Iwaniec and A. Verde, Note on the operator $\mathcal{L}(f)=f \log |f|$, Jour. of Funct. Anal., 169 (1999), 391-420.

[16] T. Iwaniec and A. Verde, A study of Jacobians in Hardy - Orlicz spaces, Proc. Roy. Soc. Edinburgh, 129(A) (1999), 539-570.

[17] G. Moscariello G., On the integrability of the Jacobian in Orlicz spaces, Math. Japonica, 40(1994), 323-329.

[18] S. Müller, A surprising higher integrability property of mappings with positive determinant, Bull. AMS, 21(1989), 245-248.

[19] S. Müller, Det $=$ det, A remark on the distributional determinant, C. R. Acad. Sci. Paris, t.311, Serie I, (1990), 13-17.

[20] S. Müller, T. Qi and B. S. Yan, On a new class of elastic deformations not allowing for cavitation, Ann. Inst. H. Poincaré Anal. Non Lineaire, 11 (2) (1994), 217-243.

[21] M. M. Rao and Z. D. Ren, Theory of Orlicz spaces, Marcel Dekker, Inc., New York (1991). 
[22] E. M. Stein, Harmonic Analysis, Princeton University Press, 1993.

Dipartimento di Matematica e Applicazioni "R. Caccioppoli"

Università degli Studi di Napoli "Federico II"

via Cintia

Napoli

Italy

(Email : anverde@unina.it) 


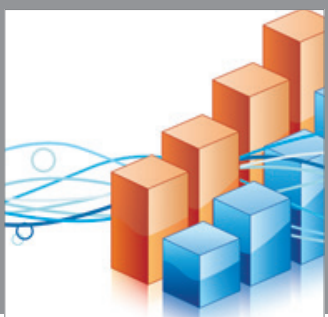

Advances in

Operations Research

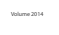

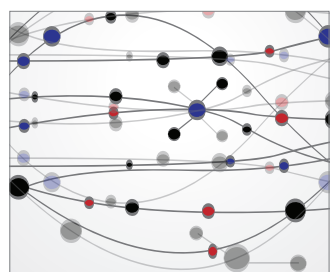

\section{The Scientific} World Journal
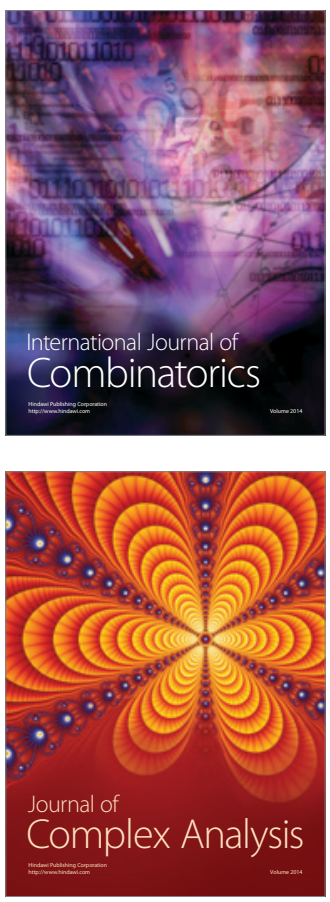

International Journal of

Mathematics and

Mathematical

Sciences
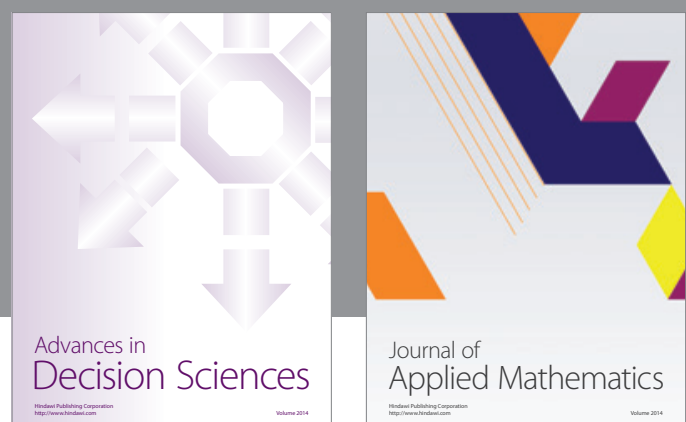

Journal of

Applied Mathematics
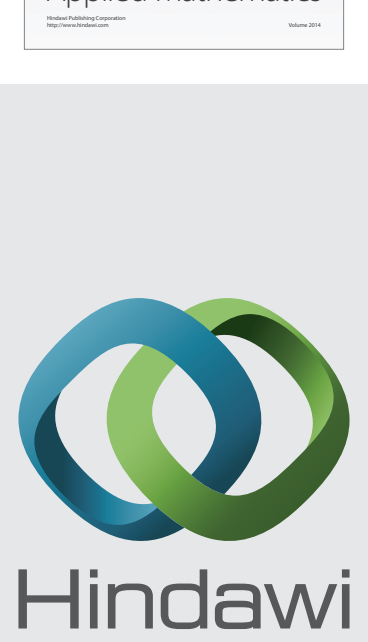

Submit your manuscripts at http://www.hindawi.com
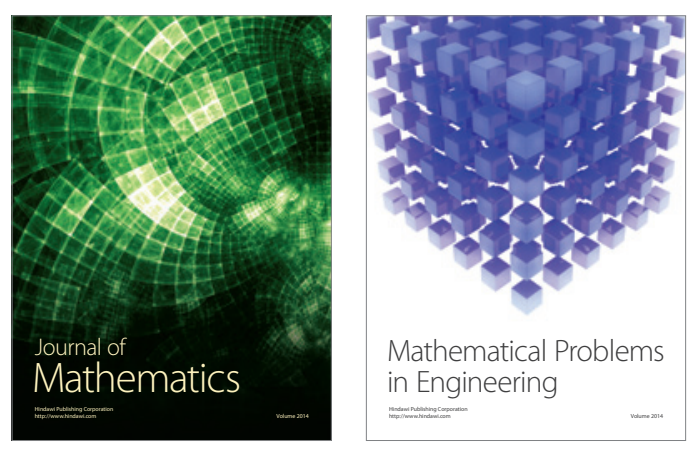

Mathematical Problems in Engineering


Journal of

Function Spaces
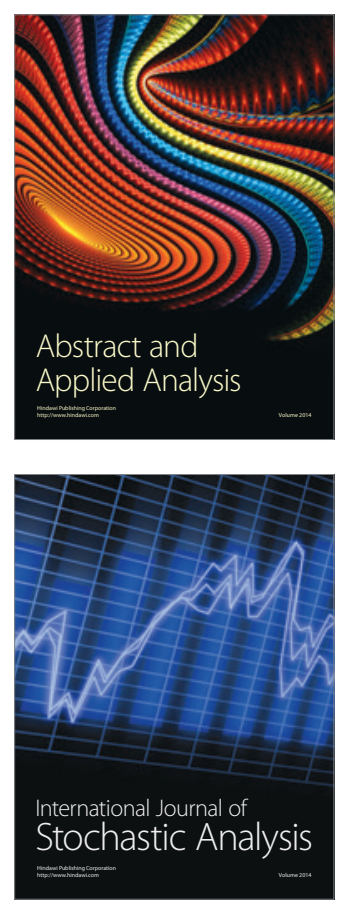

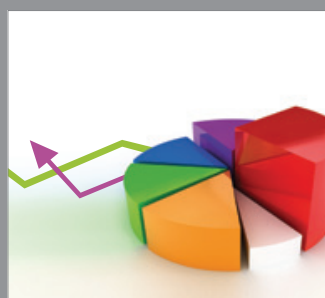

ournal of

Probability and Statistics

Promensencen
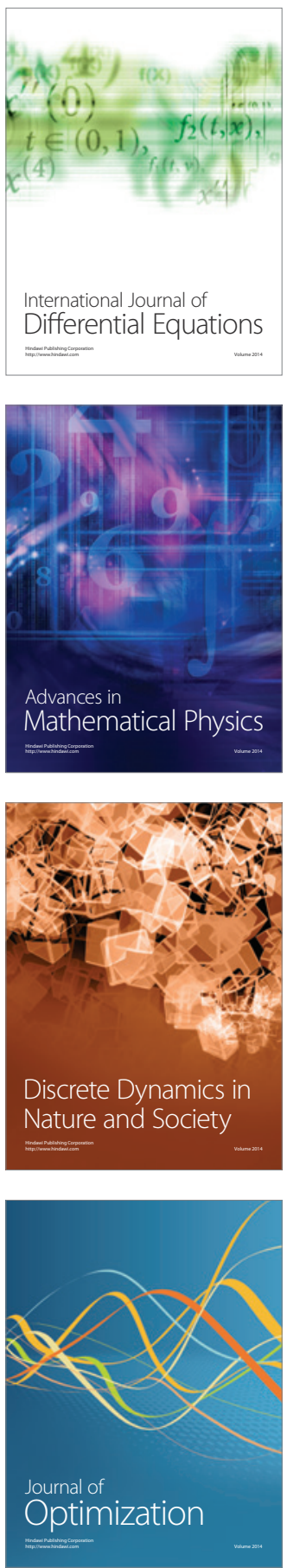